\title{
Single Mobile Micro Droplet-Particle Pairs Spatially Captured by Macro Host Droplets on a Superhydrophobic Surface
}

\author{
Gregory S. Watson, ${ }^{1}$ Bronwen W. Cribb, ${ }^{2}$ and Jolanta A. Watson ${ }^{1}$ \\ ${ }^{1}$ School of Science \& Engineering, Faculty of Science, Health, Education and Engineering, University of the Sunshine Coast, \\ Maroochydore DC, QLD 4558, Australia \\ ${ }^{2}$ Centre for Microscopy \& Microanalysis and School of Biological Sciences, The University of Queensland, \\ St. Lucia, QLD 4072, Australia
}

Correspondence should be addressed to Gregory S. Watson; gregory.watson1@jcu.edu.au

Received 12 March 2015; Revised 3 May 2015; Accepted 6 May 2015

Academic Editor: Charles Rosenblatt

Copyright (C) 2015 Gregory S. Watson et al. This is an open access article distributed under the Creative Commons Attribution License, which permits unrestricted use, distribution, and reproduction in any medium, provided the original work is properly cited.

\begin{abstract}
In this preliminary study, we demonstrate how small single water droplets can be spatially captured on the surface of individual micron sized hydrophobic coated particles $\left(\mathrm{C}_{18}\right)$ which adhere to the surface of a nonmobile larger host water droplet resting on a superhydrophobic surface. The formation of the larger droplet, particle adhesion to that droplet, and smaller droplet formation on the particle all take place spontaneously from condensation conditions. These micro droplet-particle pairs are confined to the surface (liquid-air interface) of the larger host droplet; however, they are free to engage with external forces to promote mobility. This response may find applications for particle pair transport on liquid surfaces. We also demonstrate that droplets can be captured or removed from the larger droplet surface via a self-propulsion mechanism.
\end{abstract}

\section{Introduction}

The capture, confinement, positioning, and controlled motion of micron and nanosized solid particles and droplets are of interest from both scientific and industrial perspectives. Small micron and nanosized particles can interact with liquid surfaces in a variety of ways with size, chemistry, and structure contributing to the interaction outcome. The wetting behaviour of fine particles at air-liquid surfaces is important in numerous and varied fields including soil and powder technologies as well as hair and skin care products [1].

Recent studies have explored the formation of particle films which can encapsulate a liquid forming a soft solid (liquid marble) [2-4]. These types of structures have many interesting properties and aspects such as mechanical robustness [5] and an ability to be supported above the water/marble interface [6]. Such properties make them ideal candidates for numerous applications [7-9] and interfacial studies $[10,11]$. Liquid marbles can be formed by artificial means such as depositing or rolling a drop of liquid on a layer of hydrophobic particles or via an industrial mixer but they are also found in nature $[2,3,12-14]$. A recent study has also shown the formation of these particle films via condensation methods [15].

While the studies mentioned above have focused on liquid marble formation with an emphasis on the formation and/or dynamics of marble growth, our study is primarily focused on individual solid particle-water droplet pairs formed via condensation and potential transport of the particle pair on liquid surfaces. This is in contrast to the properties of partially formed or fully formed liquid marbles. The mobility of droplets on surfaces, as well as droplet mobility driven by droplets formed via condensation, is of great interest and has been studied by others [16]. During the early stages of formation of liquid marbles, the droplet can be partially covered with particles or particle clumps. In this study, we show that partially and sparsely covered liquid marbles consisting of micro droplet-particle pairs can be formed by condensation on a superhydrophobic (SH) surface. Individual isolated hydrophobic particles (or very small clumps) are confined on larger condensed water droplets formed on the SH surface. The particles of the size 
range of $\sim 10$ microns can undergo further condensation with the formation of micro droplets. Both the particle and the droplet, which are confined by the large droplet boundary, are subject to external forces which can induce motion along the liquid-air interface.

\section{Materials and Methods}

Sectioned gecko skin (Lucasium sp.) samples were mounted on a cooled copper element maintained at a temperature below the dew point $\left(16\right.$ to $20^{\circ} \mathrm{C}$ ) and monitored with a dual temperature meter HT-L13 with thermocouples (type K) and an infrared thermometer MT300. Typical conditions of dew formation were achieved in the laboratory with humidity of $75-85 \%$ and ambient temperatures of $24-27^{\circ} \mathrm{C}$.

This work was conducted under Ethics Approval A1676 and QNP permit WITK05209908.

Droplet formation and dynamics were captured using a Nikon (V1) digital camera (video mode at 400 and 1200 frames per second (fps), $640 \times 240$ and $320 \times 120$ pixel resolution, respectively, frame rates and acquisition times of 2.50 and $0.833 \mathrm{~ms}$ per frame and video play-back at $30 \mathrm{fps}$ or $15 \mathrm{fps}$ ). The camera housing was attached to a Canon macro lens (EF-S $60 \mathrm{~mm}$ ultrasonic) for wide field and low magnification observations, while a custom made optical microscope equipped with Olympus microscope lenses (MDPlan) of 4 and 10x was utilised for higher magnifications (effectively up to $\sim 300 \mathrm{x})$.

Contamination experiments were carried out using hydrophobic carbon coated $\left(\mathrm{C}_{18}\right)$ beads, seeded by distributing a thin coating on a clean glass slide and then gently tapping the inverted slide over the sample.

\section{Results and Discussion}

The SH surface used in our study is shown in Figure S1 (in Supplementary Material available online at http://dx.doi .org/10.1155/2015/801547) and consisted of a natural surface. This surface was chosen due to the $\mathrm{SH}$ property of the topography and well defined structure. The micro structuring features small hair fibres with lengths up to $4 \mu \mathrm{m}$. The termination of the fibres can be described as spherically capped with the majority having a small radius of curvature in the range of 10 to $30 \mathrm{~nm}$. The density of hairs is very high, up to 500 per $10 \times 10 \mu \mathrm{m}$ area resulting in a submicron spacing of these fibres (Figure S1(a)). Previous studies have investigated the outer layer of such surfaces and determined the presence primarily of $\beta$-keratin $[17,18]$. X-ray Photon Electron Spectroscopy (XPS) data of our surface also indicates keratin is the key component of the surface regions where detailed scans showed binding energies of nitrogen and sulphur consistent with an organic environment.

The hair array surface supports a superhydrophobic interaction with water as shown in Figure S1(b). The apparent contact angle of water measured on the fibre array was within the range of $151-155^{\circ}$ with a contact angle hysteresis of less than 5 degrees. When we cooled the superhydrophobic surface below the dew point $\left(5-10^{\circ} \mathrm{C}\right)$, condensation formed on the surface. This consisted of small droplets typically in the range of $10-150 \mu \mathrm{m}$.

Observation of these small condensed droplets over time showed that some underwent self-propulsion, where merged droplets were propelled off the surface from the excess energy upon coalescence (see Figure S1(c) and Movie S1) [19]. This phenomenon has been observed on a number of artificial and natural surfaces where some of the excess energy, when two or more droplets merge, is converted to kinetic energy and a single droplet jumps away from the surface [20]. Due to this process, condensed droplets are not seen to grow to large dimensions on such surfaces. However, if the surface undergoes "supersaturation," the micro/nanostructures can fail to maintain the jumping droplet induced responses due to an increase in the number of nucleation sites. This can lead to "flooding" and a loss of superhydrophobicity and the formation of highly pinned Wenzel droplet morphologies [21].

In order to facilitate large droplet formation on the $\mathrm{SH}$ surface, we formed a limited number (less than 10) of defects on the surface by mechanical removal/crushing of a small number of hairs from the surface structure (less than $20 \times 20$ microns in size). This formed a defect which allowed host drops to achieve diameters of over 300 microns.

In order to produce a large water droplet with a small number of adhered particles at the air-liquid interface from condensation, we covered the $\mathrm{SH}$ surface with hydrophobic carbon coated $\left(\mathrm{C}_{18}\right)$ particles of around $10 \mu \mathrm{m}$ in diameter. This resulted in $30-45$ particles per $200 \times 200$ microns area on the SH surface. These particles exhibited low adhesion with the SH surface. Using atomic force microscopy (AFM) adhesion of individual particles (attached to AFM tipless cantilevers) to the SH surface was in the range of $10-20 \mathrm{nN}$ $\left(25^{\circ} \mathrm{C}\right.$ and $75 \%$ humidity). This low adhesion indicates that little energy is required to remove the particle from the $\mathrm{SH}$ surface.

As the surface bound condensation drop grew in dimensions around the defect region, individual hydrophobic particles were scavenged from the $\mathrm{SH}$ surface and remained adsorbed to the growing host water drop (Figure 1(a)). Merging of smaller condensates with the larger surface bound droplet was also seen to facilitate particle adsorption to the larger host drop. The strong surface flows occurring from merging events aided in this process. As seen in Figure 1, these isolated individual hydrophobic particles residing on the large host water drop could undergo secondary condensation with the formation of an isolated particle-droplet pair. The contact angle with the particle appears high as indicated by the spherical droplet shape. Other studies using $\mathrm{C}_{18}$ have measured contact angles of 105 degrees on a flat surface (e.g., [22]). This nucleation of solitary ("secondary") droplets on the particles shows an orientation away from the larger host drop and thus the particle-droplet pair is in a stable but potentially mobile configuration (e.g., Figures 1(b) and 1(c)) by virtue of the configuration and nature of particle interaction.

To investigate this mobility potential and the corresponding stability of droplets, we applied an external force generated by air currents surrounding the larger host drop (low 


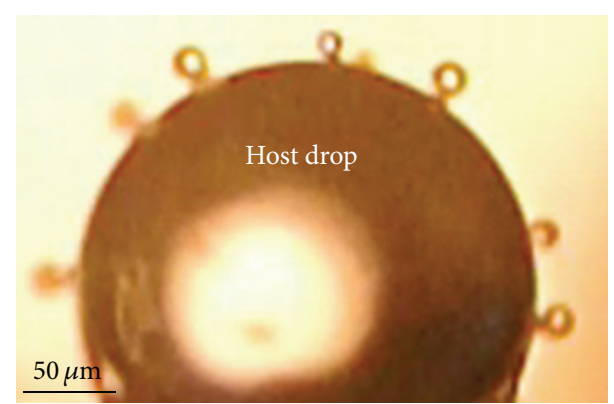

(a)

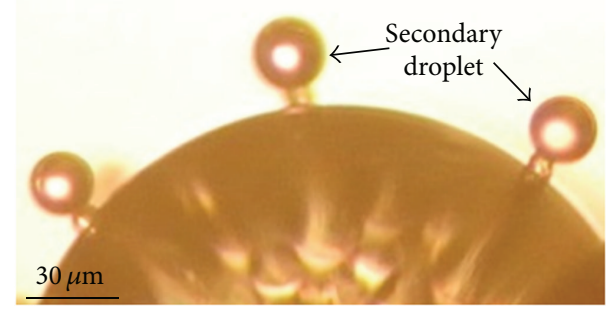

(b)

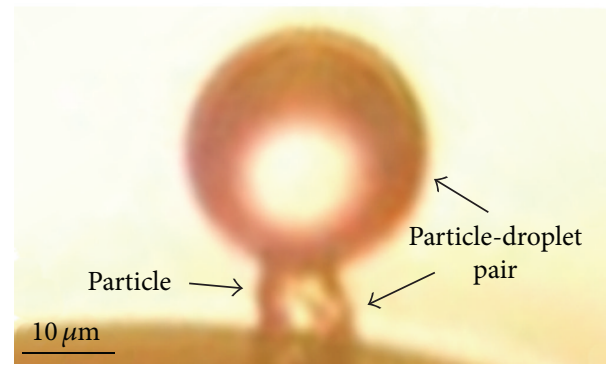

(c)

FIGURE 1: Images of small condensed micro water droplets ("secondary droplet") resting on (adhered) hydrophobic micro "particles" forming a "particle-droplet pair" which is spatially confined (at the liquid-air interface) to a larger macro droplet ("host drop"). The process occurs spontaneously from condensation conditions occurring on the particle-seeded superhydrophobic surface. (a) Optical image showing numerous particle-droplet pairs with droplet sizes typically around $30 \mu \mathrm{m}$ in diameter. (b) Higher resolution image of 3 secondary droplets residing on individual particles. (c) A single secondary droplet adhered to a particle (forming the particledroplet pair) showing the droplet nucleation site at the top of the particle.

air currents of less than 0.001 and approaching $0.05 \mathrm{~m} \mathrm{~s}^{-1}$ ). Even small moving air forces were sufficient to transport the droplet-particle pair from its initial stable location on the host drop surface (see Figure 2 and Movies S2 and S3). When we utilised a projected air current of $\sim 0.02 \mathrm{~m} \mathrm{~s}^{-1}$, we observed significant rotational motion of the particle-droplet pair around the boundary of the host drop at speeds comparable to the air flow employed (Movie S4). Interestingly, when the smaller adhered secondary droplet did make contact with the underlying SH surface, it remained fixed to the particle and adhered to the host drop. This shows that the particlesecondary droplet adhesion is higher than the secondary droplet adhesion to the SH surface and essentially confines the liquid-solid pair to the boundary of the host drop.

As the particle-droplet pairs can be transported around the surface by internal flows of the host drop or external forces, it is possible that collisions of particle-droplet pairs may occur (influenced by numerous factors such as the number of particle-droplet pairs, surface area of host drop, trajectories, speeds, and time). Observation of slow motion video showed that it is possible for two neighbouring secondary droplets upon collision to merge and self-propel off their respective particles and thus away from the host drop surface (Figure 3, Movie S5). The two particles in this case, after the merged secondary droplet has jumped away, remain separated and adsorbed to the host drop surface. This effect may be partially due to the interaction force as the secondary droplets are projected away from the particle surface (and thus the particle motion is towards the larger droplet). A number of equations have been adopted to describe the droplet propulsion when two droplets merge [23, 24]. For example, the droplet propulsion process can be viewed in a very simplified manner by considering changes in the droplet surface energies. If we consider the case where two small water droplets (not necessarily of equal size) coalesce on a superhydrophobic surface to form a larger droplet, the maximum height, $H_{\max }$, that can be attained by a droplet can be determined by integrating the velocity of a droplet over its time-of-flight resulting in the following [23]:

$$
\begin{aligned}
& H_{\max } \\
& \qquad=\frac{\rho_{w} R_{m}}{3 \rho_{a} C_{D}} \ln \left[1+\frac{9 \rho_{a} C_{D} \phi_{w}}{R_{m}^{2} \rho_{w}^{2} g}\left\{\frac{(1+f)^{2}}{\left(1+f^{3}\right)^{2 / 3}}-1\right\}\right],
\end{aligned}
$$

where $\rho_{a, w}=$ densities of air and water, respectively, $R_{m}=$ radius of the merged single droplet, $C_{D}=$ drag coefficient of droplet in air, $\phi_{w}=$ water surface tension, $g=$ gravitational acceleration $\left(9.8 \mathrm{~m} \mathrm{~s}^{-2}\right)$, and $f=$ ratio of drop diameters.

Equation (1) sets an upper limit for the maximum possible height that can be reached assuming all the released surface energy is converted into kinetic energy of the droplet and ignoring viscous forces acting within the water droplet and adhesional forces of the droplet to the surface. A more precise description of the process has been given in [24] where the authors considered the initial total kinetic energy of the coalescing droplet to include viscous forces given by

$$
E_{\text {itk }}=\Delta E_{s}-E_{\mathrm{vis}}-E_{h}-E_{w}-E_{\text {cah}},
$$

where $E_{\text {itk }}$ is the initial total kinetic energy of the coalescing droplet, $\Delta E_{s}$ is the surface energy released by the droplet coalescence, $E_{\text {vis }}$ is the viscous dissipation in the droplet, $E_{h}$ is the gravitational energy change during droplet coalescence, and $E_{w}$ is the work of adhesion. $E_{\text {cah }}$ is the energy consumed overcoming the contact angle hysteresis. The authors in that study found about $25.2 \%$ of the energy released by the droplet coalescence is converted to the effective kinetic energy in the vertical motion of the coalescing droplet jumping from the surface. In the case shown in Figure 3 (Movie S5), there is significant energy transfer to the bound 

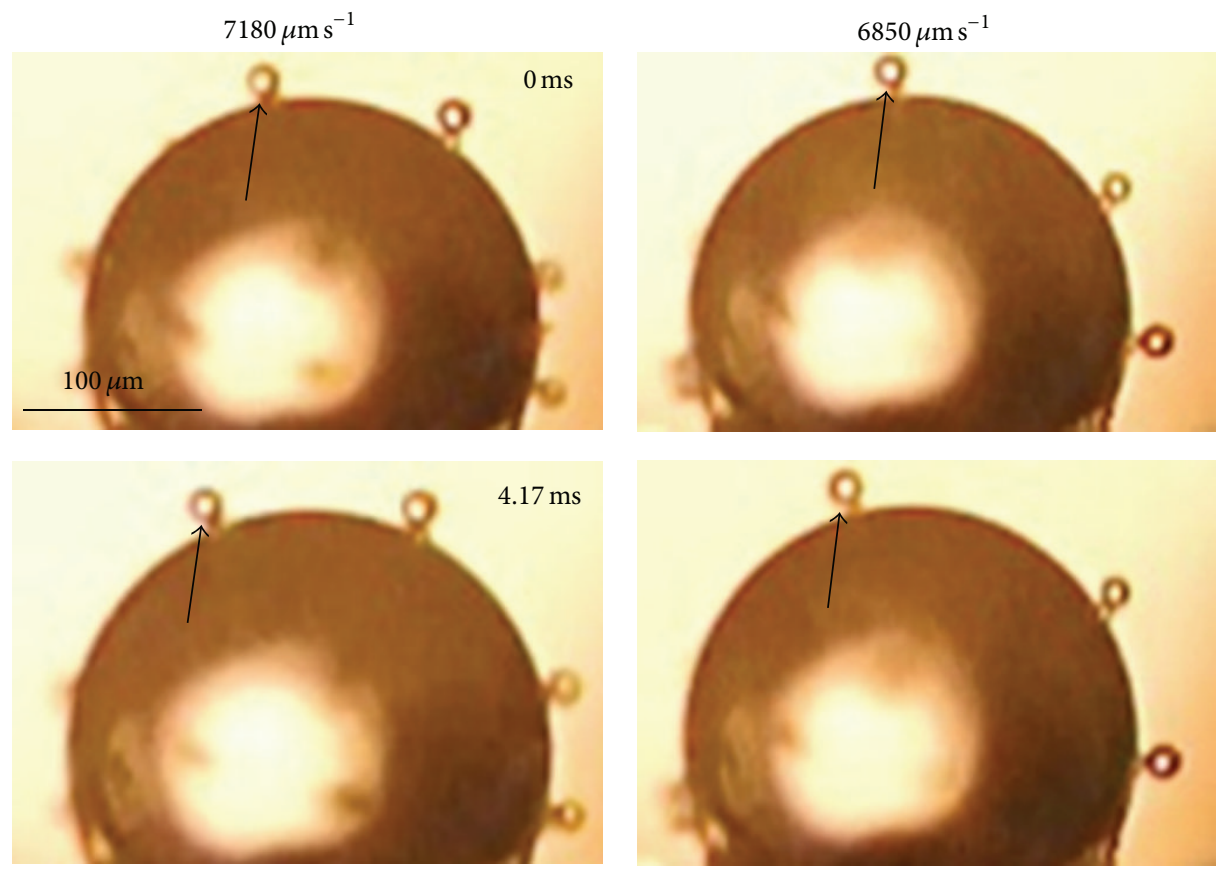

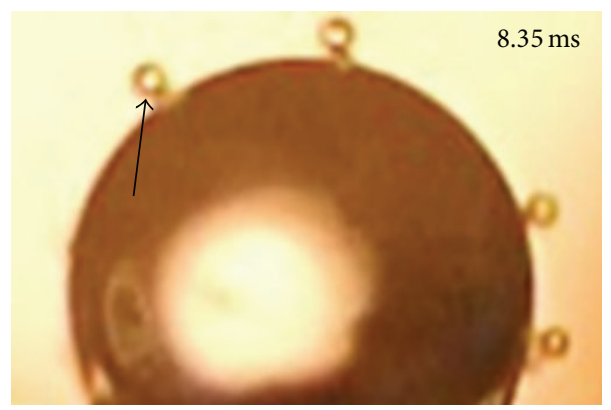

(a)

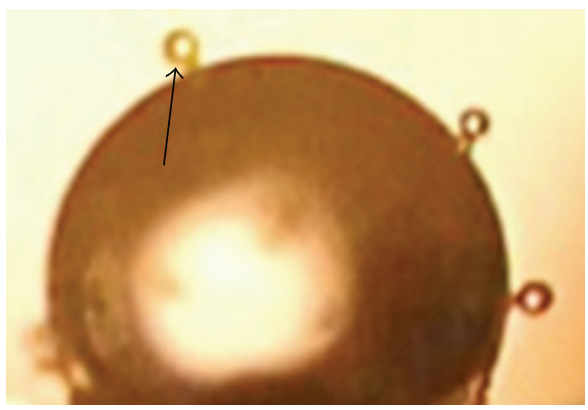

(b)

FIGURE 2: Optical images of time sequences demonstrating mobility of the particle-droplet pairs from an external air current. (a) and (b) show movement over time with particle-droplet pairs travelling at around $7000 \mu \mathrm{ms}^{-1}$ (see also Movies S2, S3, and S4).

particles and host drop as indicated by the clearly identifiable perturbations upon jumping (energy dissipation). Thus, for such a process, we could introduce two additional terms in (2) ( $E_{\text {Particle }}$, the energy loss associated with particle movement, and $E_{\text {Mdrop }}$, the energy loss associated with the macro droplet system (host drop and adhered bodies near the self-propelled location)) which will contribute to a reduction in the initial kinetic energy of the projected merged droplet:

$$
E_{\text {itk }}=\Delta E_{s}-E_{\mathrm{vis}}-E_{h}-E_{w}-E_{\text {cah }}-E_{\text {Particle }}-E_{\text {Mdrop }} \text {. }
$$

Higher frame rate acquisition of this process (and thus velocity measurements of projected particles) may yield further insights into the energy exchanges taking place; however, it is beyond the scope of this preliminary study.

Self-propulsion was also observed as a mechanism to seed the host drop with individual droplet/particles. Movie S6 illustrates this mechanism from a self-propulsion event some distance from the host drop. We have highlighted the adhered particle-droplet pair (see Figure 4(a)) coming into view shortly after contact with the host drop. In this situation, the initial impact event (Movie S6) of the small secondary droplet hitting a hydrophobic particle on the host drop and becoming fixed forms a combined particle-droplet pair. A recent study has shown that self-propulsion can remove both the secondary droplet and the adhered particle from a superhydrophobic surface [20]. Seeding of the host drop was also observed via this process.

To ascertain whether a host drop can be seeded externally to form particle-droplet pairs we seeded a larger water droplet with a small number of hydrophobic particles and then projected small secondary water droplets (simply from a spray device) onto the host drop surface. Figure 4(b) (also Movie S7) shows an example of the capture of a single secondary droplet by an individual particle via this mechanism. This process facilitated complete seeding of the particles with small secondary droplets. The secondary droplet impact on many occasions was seen to transport the then formed 


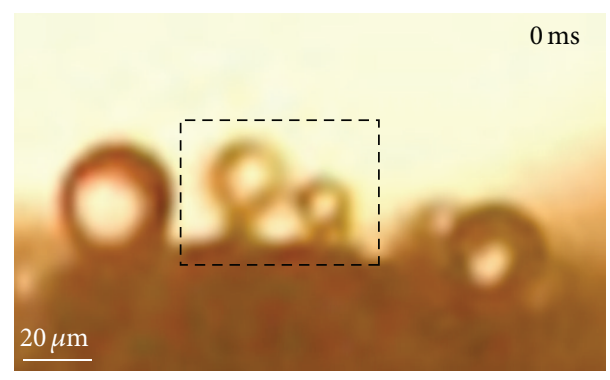

(a)

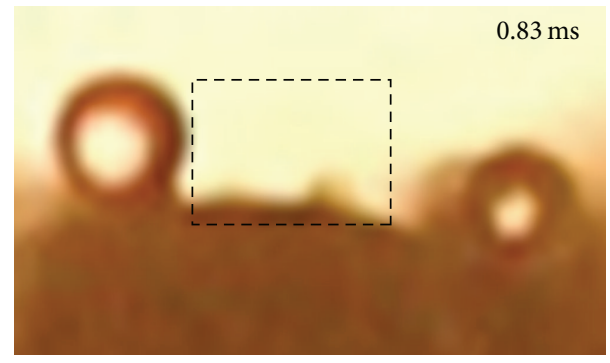

(b)

FIGURE 3: Self-propulsion of condensed secondary water droplets from the host drop. (a) Two particle-secondary droplet pairs in close proximity. (b) Less than $1 \mathrm{~ms}$ after the two secondary droplets have merged and self-propelled off the two surface bound particles. The particles remain on the surface of the host drop (see also Movie S5).

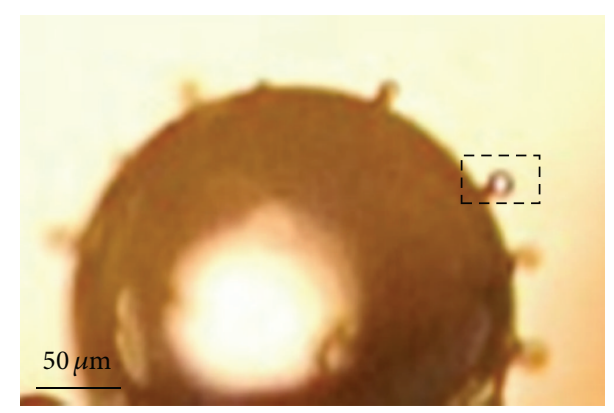

(a)
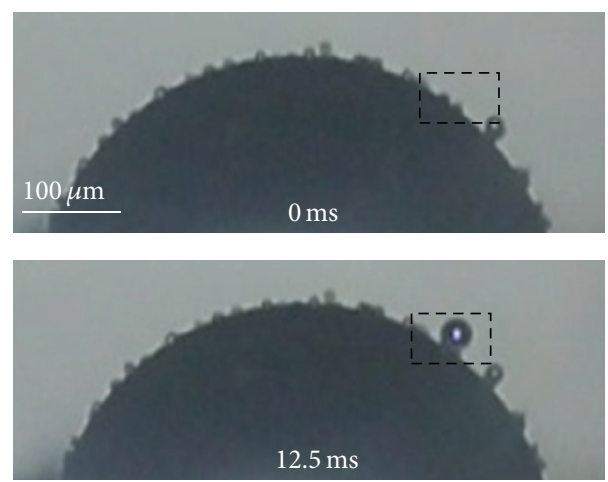

(b)

Figure 4: (a) Optical image of condensate which has previously selfpropelled onto the surface of the host drop (see also Movie S6). The image of the condensate was acquired after movement into a clear field of view (i.e., in focus). (b): before and after images of a single mobile droplet impacting onto the host drop and adhering to a single particle residing on the host drop (see also Movie S7). particle-droplet pair a significant distance along the host drop perimeter.

\section{Conclusions}

In summary, we have demonstrated the spontaneous formation of spatially confined drop-particle pairs which can undergo motional changes by internal (within larger droplet) or external forces. The controlled movement of the solidliquid particle pair around the host drop as well as selfpropulsion contributions requires further study as well as investigation of control via other external forces (e.g., hydrophobic magnetic bead-liquid droplet pair exposed to magnetic fields, mechanical oscillation/vibration). The observations of our study have applications for state-ofthe-art droplet-based smart microfluidics systems (especially involving cotransport of solid/droplet packages) and lab-onchip devices.

\section{Conflict of Interests}

The authors declare that there is no conflict of interests regarding the publication of this paper.

\section{References}

[1] N. Eshtiaghi and K. P. Hapgood, "A quantitative framework for the formation of liquid marbles and hollow granules from hydrophobic powders," Powder Technology, vol. 223, pp. 65-76, 2012.

[2] E. Bormashenko, "Liquid marbles: properties and applications," Current Opinion in Colloid and Interface Science, vol. 16, no. 4, pp. 266-271, 2011.

[3] G. McHale and M. I. Newton, "Liquid marbles: principles and applications," Soft Matter, vol. 7, no. 12, pp. 5473-5481, 2011.

[4] P. Aussillous and D. Quéré, "Liquid marbles," Nature, vol. 411, no. 6840, pp. 924-927, 2001.

[5] P. S. Bhosale, M. V. Panchagnula, and H. A. Stretz, "Mechanically robust nanoparticle stabilized transparent liquid marbles," Applied Physics Letters, vol. 93, no. 3, Article ID 034109, 2008.

[6] E. Bormashenko, Y. Bormashenko, and A. Musin, "Water rolling and floating upon water: marbles supported by a water/marble interface," Journal of Colloid and Interface Science, vol. 333, no. 1, pp. 419-421, 2009.

[7] A. V. Rao, M. M. Kulkarni, and S. D. Bhagat, "Transport of liquids using superhydrophobic aerogels," Journal of Colloid and Interface Science, vol. 285, no. 1, pp. 413-418, 2005.

[8] L. Forny, I. Pezron, K. Saleh, P. Guigon, and L. Komunjer, "Storing water in powder form by self-assembling hydrophobic silica nanoparticles," Powder Technology, vol. 171, no. 1, pp. 1524, 2007.

[9] Y. Xue, H. Wang, Y. Zhao et al., "Magnetic liquid marbles: a 'precise' miniature reactor,' Advanced Materials, vol. 22, no. 43, pp. 4814-4818, 2010.

[10] S. Fujii, S. Kameyama, S. P. Armes, D. Dupin, M. Suzaki, and Y. Nakamura, "PH-responsive liquid marbles stabilized with poly(2-vinylpyridine) particles," Soft Matter, vol. 6, no. 3, pp. 635-640, 2010. 
[11] D. Matsukuma, H. Watanabe, H. Yamaguchi, and A. Takahara, "Preparation of low-surface-energy poly[2-(perfluorooctyl)ethyl acrylate] microparticles and its application to liquid marble formation," Langmuir, vol. 27, no. 4, pp. 1269-1274, 2011.

[12] G. McHale, N. J. Shirtcliffe, M. I. Newton, F. B. Pyatt, and S. H. Doerr, "Self-organization of hydrophobic soil and granular surfaces," Applied Physics Letters, vol. 90, no. 5, Article ID 054110, 2007.

[13] N. Pike, D. Richard, W. Foster, and L. Mahadevan, "How aphids lose their marbles," Philosophical Transactions of the Royal Society B, vol. 269, no. 1497, pp. 1211-1215, 2002.

[14] M. R. Weiss, "Defecation behavior and ecology of insects," Annual Review of Entomology, vol. 51, pp. 635-661, 2006.

[15] K. Rykaczewski, J. Chinn, M. L. Walker, J. H. J. Scott, A. Chinn, and W. Jones, "Dynamics of nanoparticle self-assembly into superhydrophobic liquid marbles during water condensation," ACS Nano, vol. 5, no. 12, pp. 9746-9754, 2011.

[16] K. A. Wier and T. J. McCarthy, "Condensation on ultrahydrophobic surfaces and its effect on droplet mobility: ultrahydrophobic surfaces are not always water repellant," Langmuir, vol. 22, no. 6, pp. 2433-2436, 2006.

[17] L. Dalla Valle, A. Nardi, V. Toffolo, C. Niero, M. Toni, and L. Alibardi, "Cloning and characterization of scale $\beta$-keratins in the differentiating epidermis of geckoes show they are glycineproline-serine-rich proteins with a central motif homologous to avian $\beta$-keratins," Developmental Dynamics, vol. 236, no. 2, pp. 374-388, 2007.

[18] M. Toni, L. Dalla Valle, and L. Alibardi, “The epidermis of scales in gecko lizards contains multiple forms of $\beta$-keratins including basic glycine-proline-serine-rich proteins," Journal of Proteome Research, vol. 6, no. 5, pp. 1792-1805, 2007.

[19] J. B. Boreyko and C.-H. Chen, "Self-propelled dropwise condensate on superhydrophobic surfaces," Physical Review Letters, vol. 103, no. 18, Article ID 184501, 2009.

[20] K. M. Wisdom, J. A. Watson, X. Qu, F. Liu, G. S. Watson, and C.-H. Chen, "Self-cleaning of superhydrophobic surfaces by self-propelled jumping condensate," Proceedings of the National Academy of Sciences of the United States of America, vol. 110, no. 20, pp. 7992-7997, 2013.

[21] N. Miljkovic, R. Enright, Y. Nam et al., "Jumping-dropletenhanced condensation on scalable superhydrophobic nanostructured surfaces," Nano Letters, vol. 13, no. 1, pp. 179-187, 2013.

[22] Y.-S. Lin and V. Hlady, "Human serum albumin adsorption onto octadecyldimethylsilyl-silica gradient surface," Colloids and Surfaces B: Biointerfaces, vol. 2, no. 5, pp. 481-491, 1994.

[23] G. S. Watson, M. Gellender, and J. A. Watson, "Self-propulsion of dew drops on Lotus leaves: a potential mechanism for self cleaning," Biofouling, vol. 30, no. 4, pp. 427-434, 2014.

[24] B. Peng, S. Wang, Z. Lan, W. Xu, R. Wen, and X. Ma, "Analysis of droplet jumping phenomenon with lattice Boltzmann simulation of droplet coalescence," Applied Physics Letters, vol. 102, no. 15, Article ID 151601, 2013. 

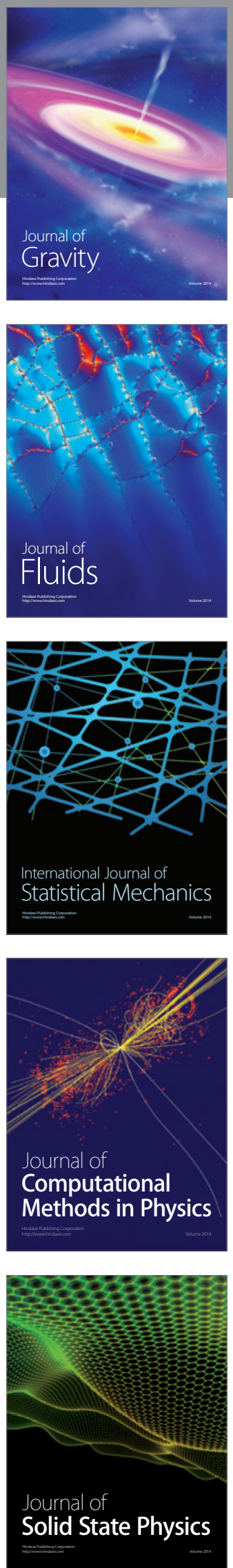

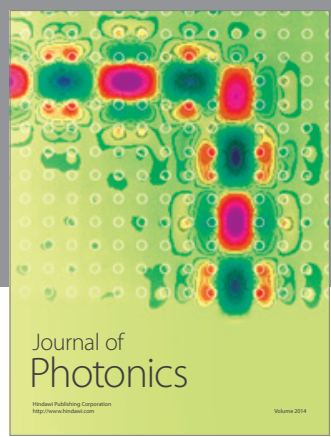

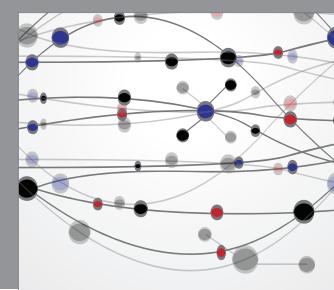

The Scientific World Journal

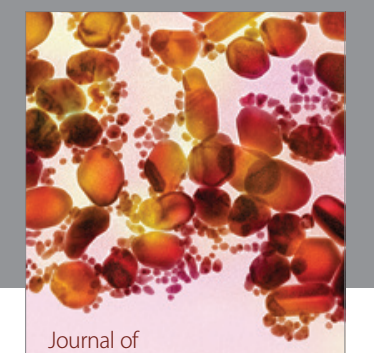

Soft Matter
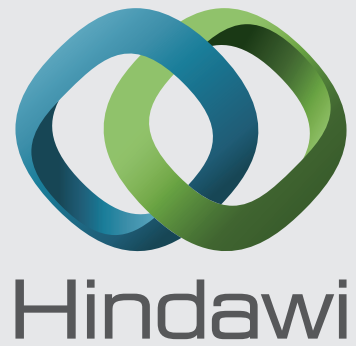

Submit your manuscripts at

http://www.hindawi.com
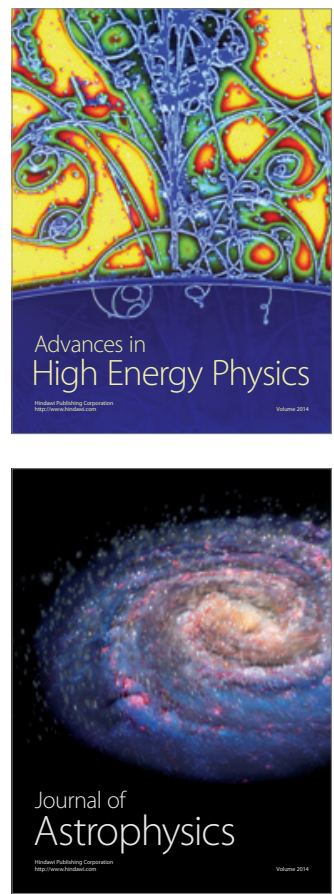
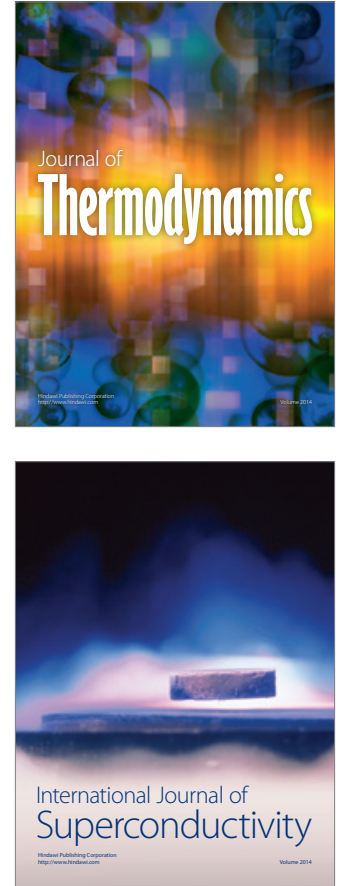
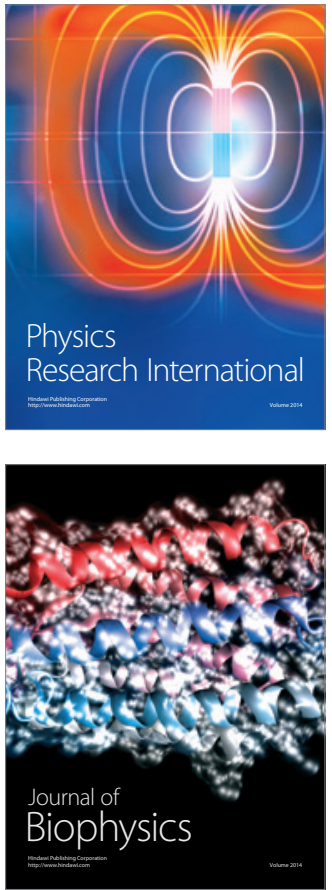
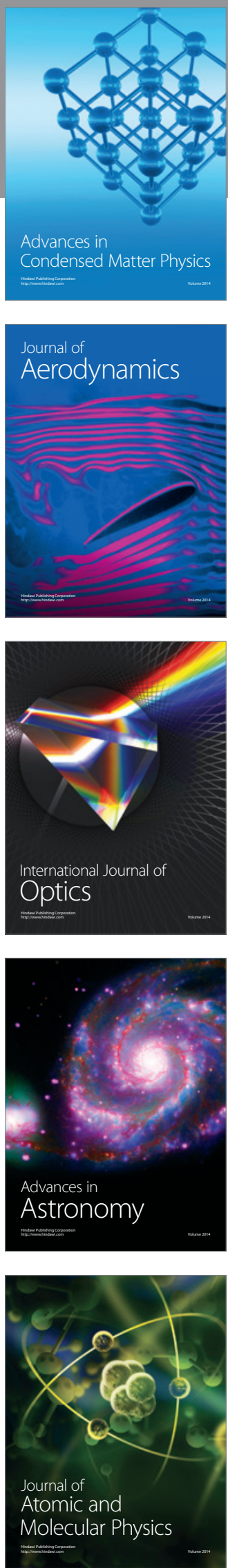